\title{
High-energy ion linacs based on superconducting spoke cavities
}

\author{
K.W. Shepard and P. N. Ostroumov \\ Physics Division, Argonne National Laboratory, 9700 S. Cass Avenue, Argonne, Illinois 60439, USA \\ J. R. Delayen \\ Thomas Jefferson National Accelerator Facility, 12000 Jefferson Avenue, Newport News, Virginia 23606, USA
}

(Received 10 June 2003; published 26 August 2003)

\begin{abstract}
The applicability of superconducting TEM-class spoke cavities to high-energy ion linacs is discussed, and detailed designs for two TEM-class, triple-spoke-loaded superconducting niobium resonant cavities are presented. The $345 \mathrm{MHz}$ cavities have a velocity range of $0.4<\beta<0.75$ and a beam aperture of $4 \mathrm{~cm}$. Spoke-loaded cavities offer several advantages compared with the higherfrequency elliptical-cell cavities that are currently being developed for this range of particle velocities. The proposed triple-spoke cavities can provide broader velocity acceptance, more accelerating voltage per cavity, reduced heat-load operation at $4.2 \mathrm{~K}$, and increased longitudinal acceptance through the high-energy section. Application to the proposed U.S. rare-isotope accelerator driver linac is discussed in detail.
\end{abstract}

DOI: 10.1103/PhysRevSTAB.6.080101

PACS numbers: $29.17 .+\mathrm{w}$

\section{INTRODUCTION}

In the first part of this paper we compare in general terms the properties of TEM-class, transmission-lineloaded resonant cavities with the properties of TM-class elliptical-cell cavities.

We then describe in some detail the design and properties of two types of three-spoke-loaded TEM-class superconducting (SC) niobium cavities [1-3] intended for use in a high-energy SC ion linac. We compare the operational characteristics of these cavities with two $805 \mathrm{MHz}$ TM-class elliptical-cell cavities, one of which has been developed for the proton driver linac for the U.S. SNS project [4] and another which is being developed for the project described below.

Finally, we examine in detail application of the spoke cavities to the high-energy section of the driver linac for the proposed U.S. rare-isotope accelerator facility (RIA) [5,6]. The RIA driver is required to produce uranium beams at $400 \mathrm{MeV}$ per nucleon and, additionally, beams of lighter ions at as high an energy as possible. To meet these requirements with a present state-of-the-art ion source, the linac must accelerate multiple-chargestate beams of the heaviest ions, which requires large longitudinal acceptance. The RIA driver must operate cw, so that minimizing $\mathrm{rf}$ losses in the linac cavities is a strong concern. As discussed below, for both of these requirements the low operating frequency of spokeloaded cavities can provide new and beneficial design options.

\section{PROPERTIES OF CAVITY GEOMETRIES}

Two main classes of SC cavity are under consideration for the medium- $\beta$ region. The first class is based on a group of several cylindrical cells operating in the cylin- drically symmetrical $\mathrm{TM}_{010}$ mode. This class is topologically identical to the one used at $\beta=1$, but with a reduced cell length [7-9]. The other class is based on singly or multiply loaded structures where each loading element supports a TEM mode. Included in this class are the types of cavities that are used extensively in the low- $\beta$ region $[10,11]$. We will refer to these two classes of cavity geometry as TM and TEM geometries, respectively. TEM and TM cavity geometries differ in three major respects: the transverse dimensions, the cell-tocell coupling, and the localization of the electromagnetic field $[12,13]$.

The transverse dimension of TM structures is of the order of $0.9 \lambda$ while for TEM structures it is of the order of $0.5 \lambda$, where $\lambda$ is the free-space wavelength of the accelerating mode. Thus, at the same frequency, TEM structures have about half the transverse size of TM structures. Alternatively, at the same transverse size, they will operate at about half the frequency. Since the BCS surface resistance of superconductors is quadratic with frequency, TEM structures operating at lower frequency will require less refrigeration and have the potential to operate at higher temperatures. Additionally, at half the frequency, a TEM structure will have half the number of cells of a TM cavity of the same length and thereby will offer a broader velocity acceptance or better efficiency in accelerating particles over a range of velocities. Operating at lower frequency also has advantages from a beam physics perspective that will be explored further in the following sections.

In TM cavities the cell-to-cell coupling takes place through the beam-line irises. In order to provide sufficient coupling, the iris opening needs to be sufficiently large. Increasing the iris diameter has two consequences: it increases the surface fields and, when the iris size 
becomes comparable to the cell length, it reduces the onaxis accelerating field for a given energy content and power dissipation, leading to a reduction of the shunt impedance of the cavity. For this reason, the cell-tocell coupling in TM cavities is often less than $2 \%$, making them sensitive to geometrical deformation and requiring accurate, tight-tolerance mechanical tuning to obtain a flat field profile. For TEM cavities, on the other hand, all the cells are strongly coupled by the rf magnetic field, with cell-to-cell coupling typically on the order of $20 \%$ to $30 \%$. Such a large cell-to-cell coupling results in a fundamental mode with large frequency spacing from any other rf modes and in a geometry that is robust with respect to mechanical tolerance. For this reason tuning of the multiple spoke cavities after fabrication in order to obtain a flat field profile is not necessary, since typical fabrication errors will not appreciably affect the field profile.

In TM structures the electromagnetic field varies slowly over position in the cavity, and the electromagnetic field level is relatively high over a large volume, resulting in a large energy content for a given accelerating gradient. In TEM cavities, on the other hand, not only is the volume smaller at a given frequency, but high electromagnetic fields are confined to the regions along the beam line and along the loading elements. This results in a lower energy content at a given frequency and gradient, which has two consequences. The first is reduced power dissipation in the cavity walls or higher shunt impedance. The second is that less $\mathrm{rf}$ power is required to phase stabilize the rf fields under the influence of microphonics-induced frequency variations.

The lower energy content of TEM cavities at a given frequency opens a possibility to design and operate a linac at lower frequency which, as discussed below, can provide numerous benefits.

There is a general perception that the peak surface electromagnetic fields at a given gradient are larger in TEM geometries than in TM geometries. Certainly this has been true historically when comparing low-velocity $(\beta<0.2)$ TEM structures with high-velocity $(\beta=1)$ TM structures. For TEM structures, however, the surface fields are relatively independent $\beta$, while for TM structures they increase significantly when $\beta$ is reduced below 1. As a consequence, at intermediate velocities, say $\beta \approx$ $0.5-0.6$, the surface fields for both types of geometries are comparable [12].

We note that surface fields may not be a dominant factor in all cases: for example, for cw linacs, rf losses may prohibit $\mathrm{cw}$ operation at gradients higher than $10 \mathrm{MV} / \mathrm{m}[14]$.

\section{TWO TRIPLE-SPOKE CAVITIES}

Designs for two SC niobium, $345 \mathrm{MHz}$, three-spokeloaded cavities for the velocity range $0.4<\beta<0.75$

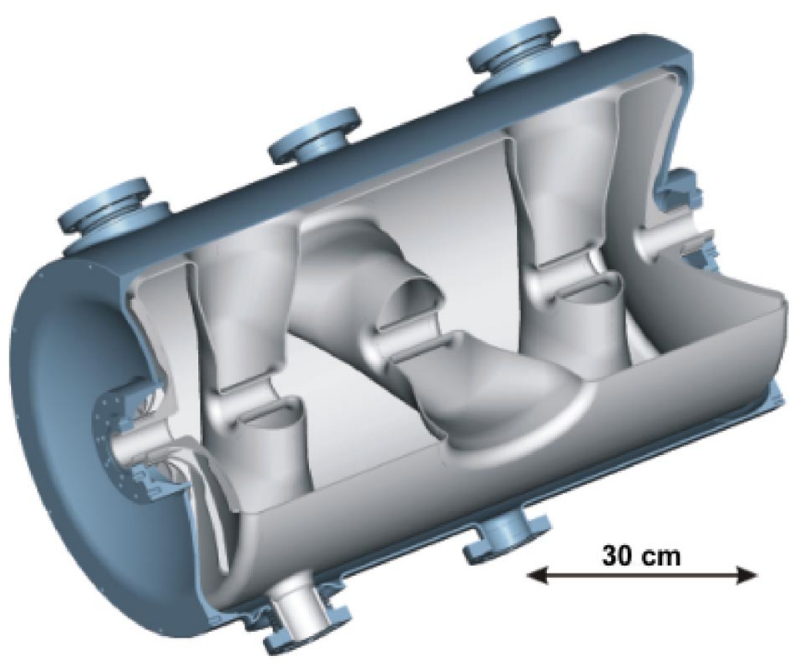

FIG. 1. (Color) Sectioned view of a $345 \mathrm{MHz}$ triple-spokeloaded cavity for $\beta_{\mathrm{GEOM}}=0.50$. The niobium cavity shell is nested in a stainless-steel jacket, which provides containment for $4.5 \mathrm{~K}$ liquid helium.

have been developed. Figure 1 shows a sectioned view of one of the two cavity types. The mechanical elements and overall design are similar to those of a recently prototyped $345 \mathrm{MHz}$ two-spoke niobium cavity [15]. The niobium elements of the cavity are formed of a $3.18 \mathrm{~mm}$ thick niobium sheet and housed in an integral stainless-steel jacket, which contains the liquid helium coolant.

In the three lowest-frequency rf eigenmodes, the threespoke elements behave as coupled, TEM-like halfwave-resonant lines. Along each spoke, the rf electric field is radial and maximum in the middle of the spoke, decreasing to zero at either end. The rf magnetic field near each spoke is circumferential, with a maximum at either end and decreasing to zero at the middle. The lowestfrequency $\mathrm{rf}$ eigenmode is the accelerating mode, in which adjacent spokes are of opposing polarity.

The structures were modeled using MICROWAVE STUDIO software [16]. A primary design objective was to minimize and balance the peak values of surface electric and magnetic fields in order to maximize the accelerating gradient as constrained by present state of the art for niobium SC cavities. Table I details the geometric parameters for the two types of three-spoke resonant cavities discussed below.

As can be seen in Fig. 1, the spoke elements are elliptical in cross section. In the central portion of the spoke, the major axis is perpendicular to the beam axis, in order to minimize the peak value of the surface electric field. As the spoke approaches the cylindrical outer wall, the elliptical section is expanded and the major axis rotates $90^{\circ}$, parallel to the beam axis, in order to minimize the peak surface magnetic field. 
TABLE I. Geometric parameters of the two types of triple-spoke cavities. The radius of the spherical end walls is $40 \mathrm{~cm}$, and all blend radii are $1.27 \mathrm{~cm}$.

\begin{tabular}{|c|c|c|c|c|c|}
\hline \multicolumn{2}{|c|}{ Geometric beta } & \multicolumn{2}{|c|}{$\beta_{\mathrm{GEOM}}=0.50$} & \multicolumn{2}{|c|}{$\beta_{\mathrm{GEOM}}=0.62$} \\
\hline \multicolumn{2}{|c|}{ Diameter of housing } & \multicolumn{2}{|c|}{$21.7 \mathrm{~cm}$} & \multicolumn{2}{|c|}{$22.9 \mathrm{~cm}$} \\
\hline \multicolumn{2}{|c|}{ Interior length of housing } & \multicolumn{2}{|c|}{$67 \mathrm{~cm}$} & \multicolumn{2}{|c|}{$85 \mathrm{~cm}$} \\
\hline \multicolumn{2}{|c|}{ Beam aperture } & \multicolumn{2}{|c|}{$4.0 \mathrm{~cm}$} & \multicolumn{2}{|c|}{$4.0 \mathrm{~cm}$} \\
\hline \multicolumn{2}{|c|}{ Elliptical spoke diameters } & End spokes & Central spoke & End spokes & Central spoke \\
\hline \multirow{2}{*}{ Central region } & Longitudinal & $5.0 \mathrm{~cm}$ & $5.6 \mathrm{~cm}$ & $6.25 \mathrm{~cm}$ & $7.4 \mathrm{~cm}$ \\
\hline & Transverse & $8.0 \mathrm{~cm}$ & $8.0 \mathrm{~cm}$ & $8.75 \mathrm{~cm}$ & $9.4 \mathrm{~cm}$ \\
\hline \multirow{2}{*}{ At cavity wall } & Longitudinal & $7.5 \mathrm{~cm}$ & $10.0 \mathrm{~cm}$ & $10.0 \mathrm{~cm}$ & $12.0 \mathrm{~cm}$ \\
\hline & Transverse & $6.5 \mathrm{~cm}$ & $5.6 \mathrm{~cm}$ & $7.0 \mathrm{~cm}$ & $7.0 \mathrm{~cm}$ \\
\hline
\end{tabular}

A crossed-spoke geometry has been chosen in which the central spoke is oriented at a right angle to the spokes at each end of the cavity. It is possible to achieve approximately the same peak surface fields with all three spokes parallel, but the parallel-spoke geometry results in the lowest-lying rf eigenmodes being very closely spaced in frequency. The crossed-spoke geometry provides a large frequency spacing and maximizes the mechanical tolerances required to balance the electric fields in the three accelerating gaps. Also, the possibility of low-level multipacting coupling two rf modes is reduced with increased mode splitting. For these reasons we have chosen the crossed-spoke geometry shown in Fig. 1.

\section{A. Electromagnetic properties}

Table II lists the principal electromagnetic parameters for the two $345 \mathrm{MHz}$ triple-spoke-loaded cavities. Included for comparison are parameters for the two $805 \mathrm{MHz}$ elliptical 6-cell cavities designed for the same particle velocities and which are part of the "baseline" design for the RIA driver linac $[6,17]$.

Figure 2 compares the velocity acceptance, i.e., the voltage gain per cavity as a function of particle velocity,

TABLE II. Electromagnetic parameters for the two triplespoke cavities compared with two elliptical-cell 6-cell cavities of similar geometric $\beta=v / c$ developed for the SNS linac and for the RIA driver linac.

\begin{tabular}{lcccc}
\hline \hline \multicolumn{1}{c}{ Cavity type } & $\begin{array}{c}\text { Triple } \\
\text { spoke }\end{array}$ & $\begin{array}{c}\text { Elliptical } \\
6 \text { cell }\end{array}$ & $\begin{array}{c}\text { Triple } \\
\text { spoke }\end{array}$ & $\begin{array}{c}\text { Elliptical } \\
6 \text { cell }\end{array}$ \\
\hline Beta geometric & 0.50 & 0.47 & 0.62 & 0.61 \\
Frequency $(\mathrm{MHz})$ & 345 & 805 & 345 & 805 \\
Length $(\mathrm{cm})$ & 65.2 & 52.55 & 80.87 & 68.2 \\
$\mathrm{G}(\Omega)$ & 85.7 & 136.7 & 93.0 & 179.0 \\
$R / Q=V^{2} / P Q(\Omega)$ & 494 & 160 & 520 & 279 \\
$\quad \mathrm{At}$ an accelerating gradient of & $1 \mathrm{MV} / \mathrm{m}:$ \\
rf energy $(\mathrm{mJ})$ & 397 & 341 & 580 & 330 \\
Peak $E$ field $(\mathrm{MV} / \mathrm{m})$ & 2.88 & 3.41 & 2.97 & 2.71 \\
Peak $B$ field $(\mathrm{G})$ & 86.5 & 69 & 88.6 & 57.2 \\
\hline \hline
\end{tabular}

of the triple-spoke and elliptical-cell cavities. The accelerating field level is determined by setting the peak surface electric field at $27.5 \mathrm{MV} / \mathrm{m}$ for all the cavities, the feasibility of which is indicated by recent experimental results for a number of niobium spoke and ellipticalcell cavities [15,18-20].

Note that, because of the lower frequency enabled by using a TEM structure, the triple-spoke geometry provides significantly broader velocity acceptance and higher voltage gain than the elliptical-cell option.

The breadth of velocity acceptance varies inversely with the number of cells (each of length $\beta \lambda / 2$ ) incorporated into the accelerating structure. The more cells, the more voltage gain per cavity, but the narrower the velocity acceptance. In the present case, for a given particle velocity, the cell length is a factor of 2.33 longer for the $345 \mathrm{MHz}$ three-cell spoke cavities, which can therefore provide at the same time both more voltage and broader velocity acceptance than an $805 \mathrm{MHz}$ sixelliptical-cell cavity. As discussed below, using these two triple-spoke cavities we can span the required velocity

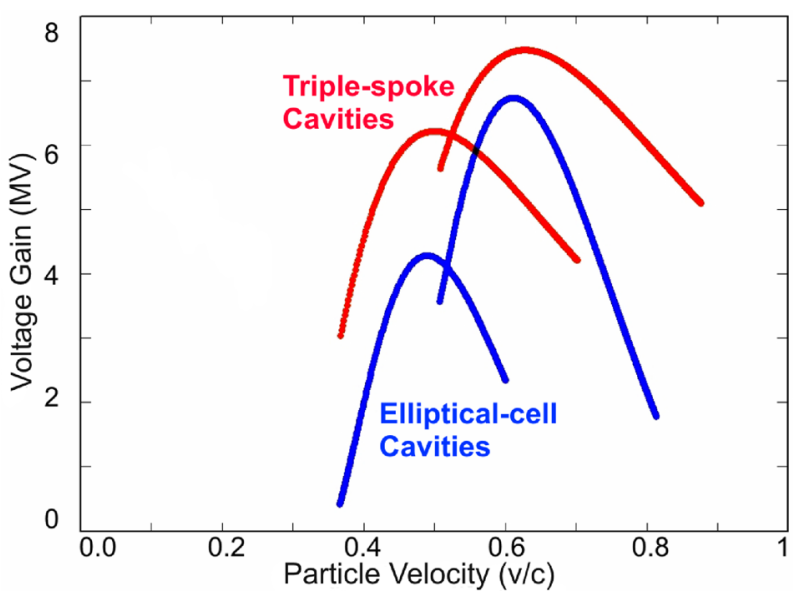

FIG. 2. (Color) Voltage gain per cavity as a function of particle velocity for two $345 \mathrm{MHz}$ triple-spoke cavities compared with two $805 \mathrm{MHz}$ elliptical 6-cell cavities, all operating at a peak surface electric field of $27.5 \mathrm{MV} / \mathrm{m}$. 
range not only with fewer cavities, but also with fewer types of cavity.

\section{B. Cavity rf heat load}

The excellent shunt impedance of the spoke-loaded cavities, together with the relatively low operating frequency, enables us to design the linac not only to reduce the $\mathrm{rf}$ heat load but also to operate at $4.2 \mathrm{~K}$.

Operation at $4.2 \mathrm{~K}$ is impractical for the ellipticalcell cavities which, because of the very much higher surface resistance at $805 \mathrm{MHz}$, must operate at $2 \mathrm{~K}$. The lower-frequency TEM spoke cavities can be operated at $4.2 \mathrm{~K}$, which eliminates the need for subatmospheric operation, substantially simplifying the cryogenic system. The refrigerator, distribution system, and linac cryostats would all be appreciably simpler to build, maintain, and operate at a temperature of $4.2 \mathrm{~K}$ as opposed to $2 \mathrm{~K}$.

Figure 3 shows the rf heat load in watts per MV of accelerating potential for the $345 \mathrm{MHz}$ triple-spoke cavities operating at $4.2 \mathrm{~K}$ (solid lines). For comparison, we show the refrigeration load for the $805 \mathrm{MHz}$ elliptical 6-cell cavities operating at $2 \mathrm{~K}$ (dashed lines). The $2 \mathrm{~K}$ system is assumed to achieve an efficiency such that a $0.25 \mathrm{~W}$ into $2 \mathrm{~K}$ is equivalent to $1 \mathrm{~W}$ of heat load into $4.2 \mathrm{~K}$ [21,22]. The heat load in Fig. 3 is estimated assuming operation of all cavities at a peak surface electric field of $27.5 \mathrm{MV} / \mathrm{m}$, as discussed above.

In Fig. 3, the heat load is shown as a function of cavity performance specified in terms of an empirically determined residual resistance characterizing the quality of the SC cavities. The residual resistance $R_{\mathrm{EXC}}$ is taken to be the resistance required to be added to the

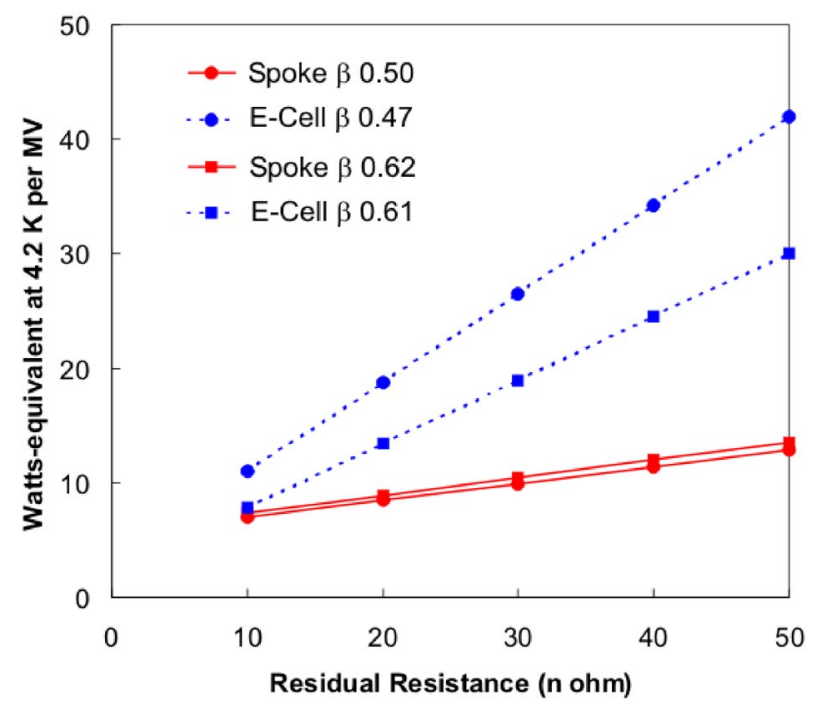

FIG. 3. (Color) A rf load into helium refrigeration as a function of cavity performance for operation at a peak surface electric field of $27.5 \mathrm{MV} / \mathrm{m}$. See text for details.
BCS SC surface resistance $R_{\mathrm{BCS}}$ [23] to account for the measured total rf loss $\left(R_{\mathrm{EXC}}+R_{\mathrm{BCS}}\right)$ at a given temperature and field level. Design goals recently quoted for elliptical-cell cavities range over $25<R_{\mathrm{EXC}}<50 \mathrm{n} \Omega$ for long-term, on-line performance $[18,20]$. At this performance level, the use of spoke-loaded cavities would reduce the refrigeration load per MV of linac by a factor of 2 or more relative to the higher-frequency ellipticalcell alternative.

Note that for the spoke cavities, the reduced slope of heat load as a function of residual resistivity provides a large design margin in terms of overall linac heat load as contingent on a range of SC cavity performance.

\section{LOW-FREQUENCY CAVITIES AND REDUCED BEAM LOSS}

Medium-energy high-power ion linacs, both those presently operating and those that are being constructed, use room-temperature drift-tube accelerating structures for velocities less than $0.6 c$ [4,24-27]. As is well known, the shunt impedance of drift-tube structures becomes low at velocities above $0.3 c$. At lower velocities, lowerfrequency structures are more efficient. In order to minimize the construction costs of a linac and to keep the operating efficiency high, the high-energy section (above $0.4 c$ ) is usually operated at a higher harmonic of the bunch frequency. The resultant frequency jump abruptly reduces the phase width of the stable area in longitudinal phase space and as a consequence reduces the longitudinal acceptance.

The reduced acceptance can be a problem in highpower machines because of beam halo. Downstream of the frequency transition, some halo particles are likely to be located outside of the longitudinally stable area. In the velocity range below $\sim 0.55-0.6 c$ the focusing lattice usually provides stable motion only for the particles inside the separatrix. Halo particles outside of the separatrix can become unstable transversely and are then lost in the high-energy section of the linac. This mechanism can be a major source of beam loss in high-power linacs.

If, however, the frequency jump in ion linacs takes place above velocities $\sim 0.6 c$, a wide range of particle momentum will be stable transversely and can be transported to the end of the linac even if particles are located outside of the longitudinally stable area. At sufficiently high velocities, transverse focusing is stable for a wide range of particle momentum due to the significant suppression of the defocusing term of the accelerating field. In this case, the lower energy particles can be transported all the way through the linac and successfully dumped in a high dispersion area of the switchyard.

Different high-power machines have different mechanisms of halo formation. For example, in proton $/ \mathrm{H}^{-}$ linacs the main source of halo formation is the beam 
space charge. In the case of the RIA driver linac discussed below, the main source of beam halo is scattering in the final, relatively thick stripping foil.

\section{SPOKE-CAVITY HIGH-ENERGY SECTION FOR THE RIA DRIVER LINAC}

The proposed U.S. RIA facility requires a driver linac producing cw, $100 \mathrm{~kW}$ ion beams at energies of $400 \mathrm{keV} /$ nucleon or more for all ion species. A baseline design for the driver linac has been developed which consists of an array of several types of independently phased, short, SC accelerating structures $[6,17]$. In order to produce sufficiently intense beams the linac must accelerate multiple-charge state beams of the heavier ions. This requirement largely drives the lattice design and beam dynamics for the linac.

We confine our discussion to the high-energy section of the linac which, for uranium beams, is between the final charge stripping at $80 \mathrm{MeV} /$ nucleon and the output at $400 \mathrm{MeV} /$ nucleon. The high-energy section comprises approximately $2 / 3$ of the overall linac, providing $780 \mathrm{MeV}$ of acceleration. We compare two options: the baseline design, which employs three types of elliptical-cell 6cell cavities, and a design using the two types of triplespoke-loaded cavity described above, i.e., $350 \mathrm{MHz}$, $\beta_{G}=0.5$ and $\beta_{G}=0.62 c$. In what follows we refer to the first option as the elliptical-cell linac (ECL) and the second option as the triple-spoke linac (TSL).

The cavity array is determined by the requirement to produce uranium beams at $400 \mathrm{MeV} /$ nucleon. The uranium beam at the entrance to the high-energy section is at an energy of $81 \mathrm{MeV} /$ nucleon and is composed of four charge states. Table III shows the number and types of cavities required, for each of the two design options, in order to accelerate the uranium beam to an energy of $400 \mathrm{MeV} /$ nucleon. The TSL option requires substantially fewer cavities than the ECL design.
TABLE IV. Output beam energies ( $\mathrm{MeV} /$ nucleon) for several ion species for the two design options.

\begin{tabular}{ccc}
\hline \hline Species & Triple spoke & Elliptical cell \\
\hline$H$ & 956 & 908 \\
$\mathrm{He}^{3}$ & 712 & 713 \\
$D$ & 574 & 588 \\
$A^{40}$ & 517 & 537 \\
$\mathrm{Xe}^{136}$ & 442 & 460 \\
$\mathrm{U}^{238}$ & 405 & 404 \\
\hline \hline
\end{tabular}

For lighter ions, the charge to mass ration is higher, and the cavity array can be tuned for higher beam energy, as shown in Table IV. One effect of the broader velocity acceptance of the triple-spoke cavities is to produce an appreciably higher-energy proton beam than the ECL design.

A focusing system with room-temperature quadrupole doublets located outside of the cryostat is used in the ECL "benchmark" design and could be used for the TSL. We have chosen, however, to use $50 \mathrm{~cm}$ long, $9 \mathrm{~T}$ SC solenoids in the TSL design. The solenoids provide strong transverse focusing for all ion beams and can be incorporated into the cryostats, economizing space along the beam line.

A realistic accelerating-focusing lattice for the TSL high-energy section is based on two types of cryostats, as shown in Fig. 4. The first type is $3.4 \mathrm{~m}$ in length and contains one SC solenoid and three $\beta_{G}=0.5$ triple-spoke cavities. The second type is $5.3 \mathrm{~m}$ in length and contains one solenoid and four $\beta_{G}=0.62$ triple-spoke cavities. The space between the cryostats, approximately $0.5 \mathrm{~m}$, is adequate for beam diagnostics and beam steering magnets. We note that the latter could be integrated into the solenoids inside the cryostat.

Table V summarizes and compares some of the principal parameters and features of the TSL and the

TABLE III. SC cavity arrays required for the high-energy section for the RIA driver linac for the two design options considered.

\begin{tabular}{|c|c|c|c|c|c|c|}
\hline Cavity type & $\beta$ geometric & $\begin{array}{l}\text { Frequency } \\
\quad(\mathrm{MHz})\end{array}$ & $\begin{array}{l}\text { Length } \\
(\mathrm{cm})\end{array}$ & $\begin{array}{c}E_{\mathrm{PEAK}} \\
(\mathrm{MV} / \mathrm{m})\end{array}$ & $\begin{array}{c}E_{\mathrm{ACC}} \\
(\mathrm{MV} / \mathrm{m})\end{array}$ & $\begin{array}{c}\text { Number of } \\
\text { cavities }\end{array}$ \\
\hline \multicolumn{7}{|c|}{ Triple-spoke cavity option } \\
\hline 3 spoke & 0.50 & 345.0 & 65 & 27.5 & 9.5 & 42 \\
\hline 3 spoke & 0.62 & 345.0 & 81 & 27.5 & 9.3 & 98 \\
\hline \multicolumn{6}{|c|}{ Total no. of cavities $=$} & Elliptical-cell 6-cell cavity option \\
\hline 6 cell & 0.47 & 805.0 & 55 & 27.5 & 8.1 & 60 \\
\hline 6 cell & 0.61 & 805.0 & 68 & 27.5 & 9.9 & 88 \\
\hline 6 cell & 0.81 & 805.0 & 91 & 27.5 & 12.6 & 32 \\
\hline \multicolumn{6}{|c|}{ Total no. of cavities $=$} & 180 \\
\hline
\end{tabular}




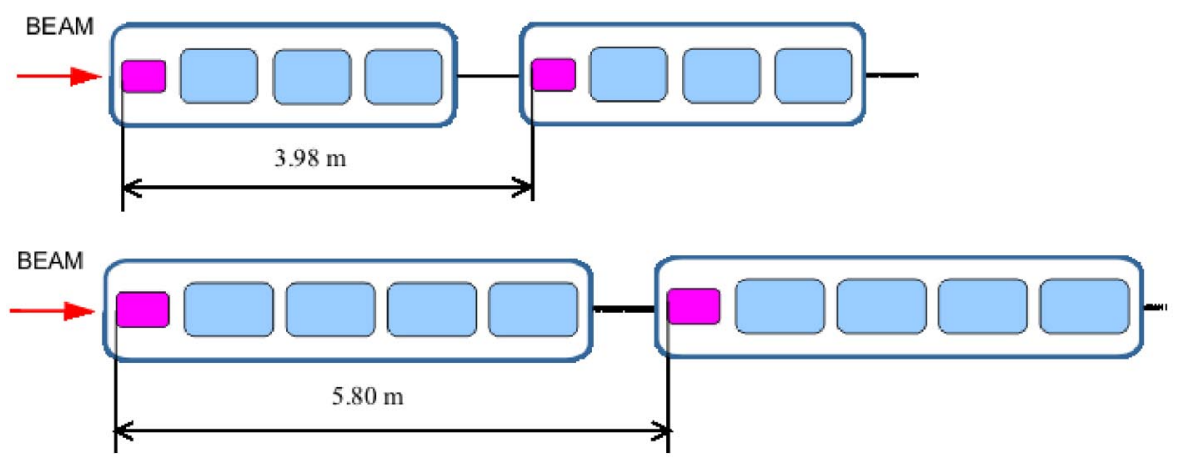

FIG. 4. (Color) Layout of the cryostats containing two types of triple-spoke-loaded superconducting cavity: $\beta_{G}=0.5$ (top) and $\beta_{G}=0.62$ (bottom).

TABLE V. Summary of the parameters for the two design options for the high-energy section of the RIA driver linac.

\begin{tabular}{lcc}
\hline \hline \multicolumn{1}{c}{ Parameter } & $\begin{array}{c}\text { Triple } \\
\text { spoke }\end{array}$ & $\begin{array}{c}\text { Elliptical } \\
\text { cell }\end{array}$ \\
\hline Frequency $(\mathrm{MHz})$ & 345 & 805 \\
Peak $E$ field $(\mathrm{MV} / \mathrm{m})$ & 27.5 & 27.5 \\
No. of cavity types & 2 & 3 \\
Total no. of cavities & 140 & 180 \\
Temperature $(\mathrm{K})$ & 4.2 & 2 \\
Aperture $(\mathrm{mm})$ & 40 & 80 \\
Synchronous phase & -25 & -30 \\
\multicolumn{1}{l}{$\quad$ Normalized acceptance } & \\
Transverse $(\pi \mathrm{mm}$ mrad) & 35 & 70 \\
Longitudinal $(\pi \mathrm{keV} / \mathrm{unsec})$ & 280 & 60 \\
\hline \hline
\end{tabular}

ECL design options for the RIA driver linac high-energy section.

\section{A. Beam dynamics}

Beam dynamics were studied and compared for both design options by numerical modeling using the code TRACK [28], which integrates the particle motions through 3D electromagnetic fields. The 3D rf electromagnetic fields were obtained using the electrodynamics code CST MICROWAVE STUDIO (version 4.0). Both transverse and longitudinal rms beam sizes were carefully matched prior to detailed beam dynamics simulation.

The numerical simulation starts at the entrance of SC linac $(\beta=0.02)$ with a two-charge-state beam from the RFQ injector [29]. Multiple-charge state transport systems follow each of the two strippers. For the uranium beam, the low-beta section accelerates two charge states, $28+$ and $29+$, to the first stripper and the medium beta section accelerates five charge states to the second stripper. After the second stripper, the four charge states 88-91+ are transported to the entrance of the highenergy section.

In the simulations, the synchronous phase for the ECL is set at $-30^{\circ}$, while in the TSL it can be reduced to $-25^{\circ}$ because of the large longitudinal acceptance of the lowerfrequency cavity array.

The simulation results shown are for $10^{4}$ particles per bunch and include 30 seeds of random errors both in alignment of all elements ( $300 \mu \mathrm{m})$, and also in the phase and amplitude of the electromagnetic fields in the SC cavities. The latter errors were set at a maximum of $\pm 1^{\circ}$ and $\pm 1 \%$ in the lowest-frequency resonant cavities $(58 \mathrm{MHz})$, and of $\pm 0.5^{\circ}$ and $\pm 0.5 \%$ in all higher-frequency resonators.

Figures 5 and 6 show the transverse and longitudinal beam envelopes, respectively, through the high-energy section of the triple-spoke cavity based linac. The envelopes contain all four charge states. Both maximum size and rms sizes are plotted.

In Fig. 7 the longitudinal acceptance for both the ECL and TSL designs, determined by Monte Carlo simulations, is outlined with blue dots. The red areas in Fig. 7 show, for both design options, the longitudinal phase space of the four-charge-state uranium beam at the entrance of the high-energy section. The TSL design increases the longitudinal acceptance by a factor of 4.7 and, as discussed below, substantially the margin for avoiding beam loss.

For the ECL design, the elliptical-cell iris diameter is set at $80 \mathrm{~mm}$ in order to provide adequate cell-cell coupling. Such a large aperture provides a very large transverse acceptance for this design option. The $40 \mathrm{~mm}$ beam aperture of the triple-spoke cavities provides, however, an ample safety factor in the transverse plane. Indeed, the aperture-to-rms-beam-size ratio, a critical factor for high-power linacs, remains in the range 1620 throughout the TSL linac.

We note that the possibilities for beam loss are likely to be much more strongly impacted by the longitudinal rather than the transverse acceptance, since the situation is more marginal in longitudinal phase space [30]. While the numerical studies reported to date indicate that both the baseline ECL and the TSL design options can provide good beam quality for multiple-charge-state beams, the reported studies have not included detailed simulation of 


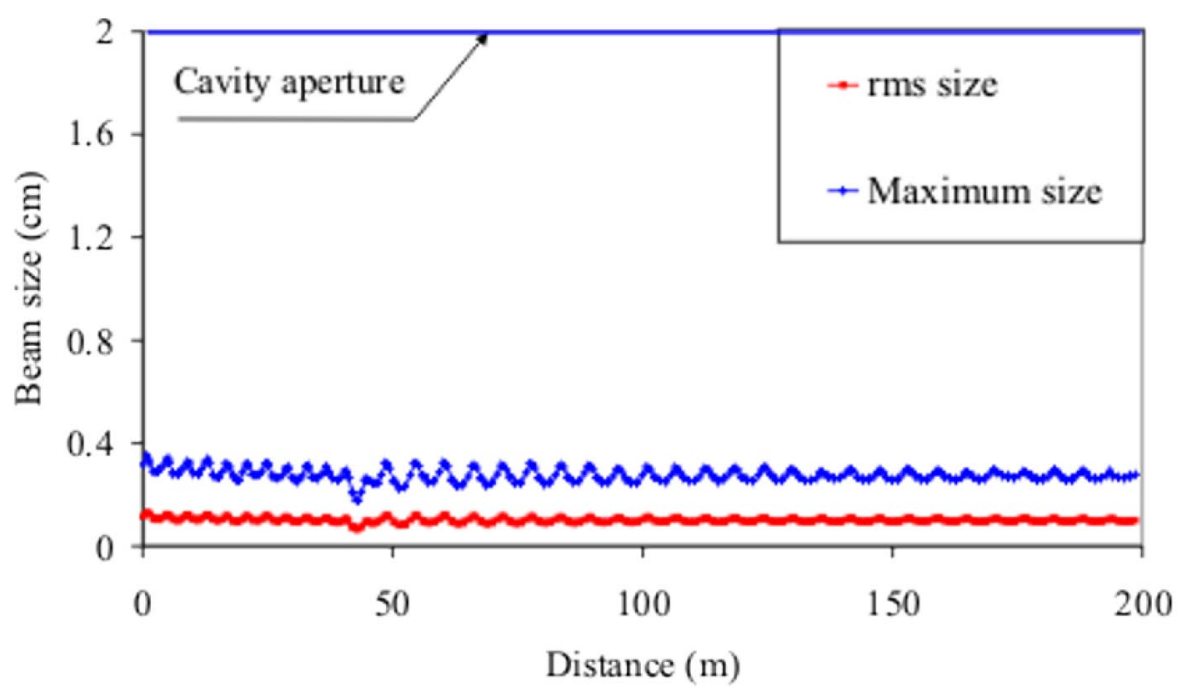

FIG. 5. (Color) Beam in the transverse horizontal plane through the high- $\beta$ section of the triple-spoke cavity based linac. The upper limit of the scale is the cavity aperture, the lower curve is the rms size, and the upper curve the maximum size.

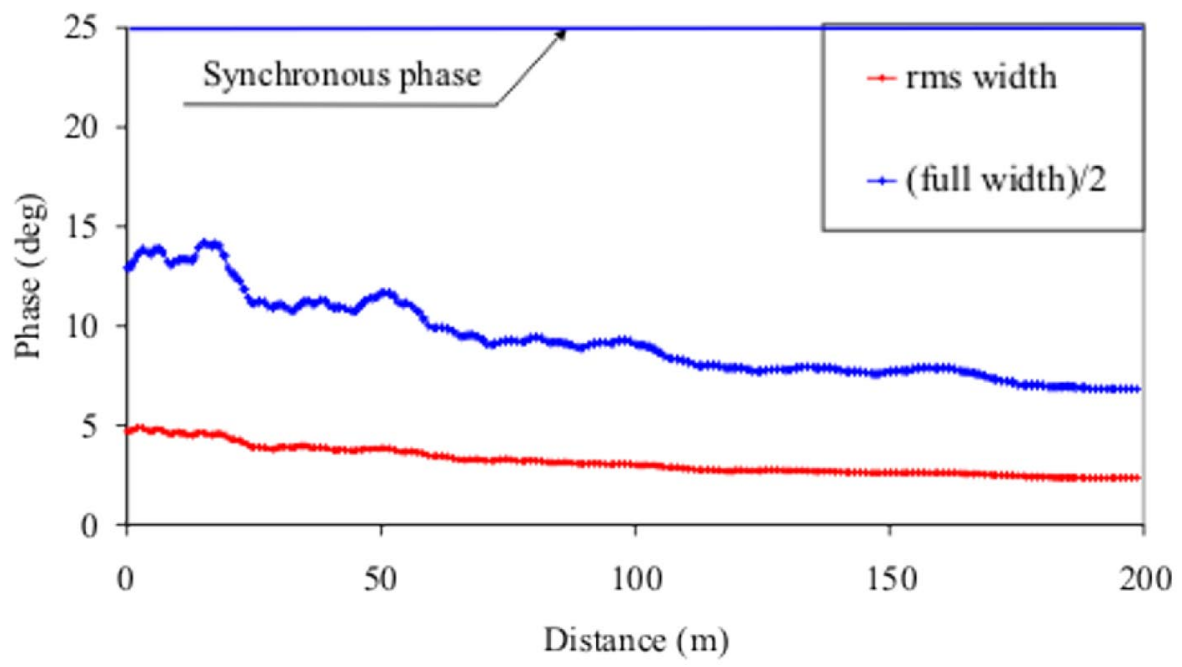

FIG. 6. (Color) Beam size in the longitudinal plane through the high- $\beta$ section of the triple-spoke cavity based linac. The lower curve is the rms size and the upper the maximum size.

the effects of scattering in the high-energy stripping foil required for the heaviest ions.

\section{B. Effects of stripping on beam halo}

At the present time, experimental data characterizing the stripping process for high-power heavy-ion beams at energies $\sim 85 \mathrm{MeV} / u$ are not available. We note, however, that recent calculations for EURISOL [31] indicate the possibility of as much as several parts in $10^{4}$ of the beam exhibiting extreme energy straggling on the low-energy side. Because of the multiple-charge state nature of the beam, it would be difficult to clean such a low-energy tail in the chicane section following the stripper.

The beam emittance shown in Fig. 7 was obtained by, first, simulating the scattering in the foil numerically using the SRIM code [32]. Second, we assume the stripping foil to fluctuate in thickness by $\pm 2.5 \%$ and further assume the fluctuation to be small scale and uniformly distributed. Such fluctuations are caused by thermal deformations and by beam-induced sputtering of the foil itself. Thickness fluctuations contribute substantially to the emittance growth because the total energy loss is large (3.29 MeV/u). The resultant simulated foil scattering produces distributions which are roughly Gaussian [30] with standard deviations in energy of $17.6 \mathrm{keV} / u$, and in angle of $0.5 \mathrm{mrad}$. The above results were used to regenerate the particle coordinates just after the stripper, from which point on the beam was simulated using the TRACK code.

As was discussed in the previous section, a good method to avoid beam loss from the low-energy tail is 

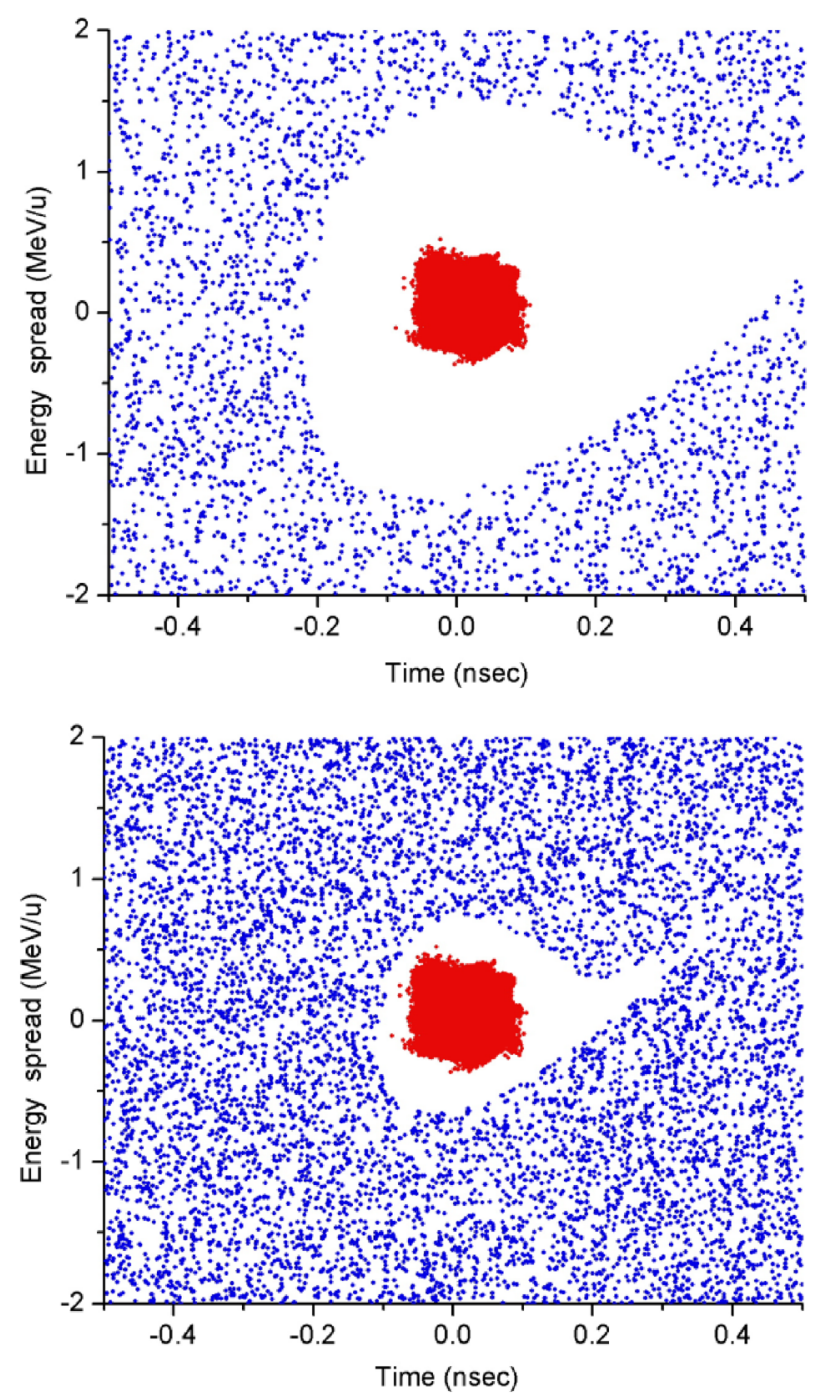

FIG. 7. (Color) Longitudinal phase space at the entrance to the high-energy section. A four-charge-state uranium beam is shown in red, superimposed on a scatter plot (blue) showing the longitudinal acceptance.

to provide large longitudinal acceptance in the high-energy section. The TSL design option achieves this to a much greater extent than the baseline ECR design, which more than doubles the linac frequency right at the second stripper.

\section{CONCLUSIONS}

TEM-class, spoke-loaded superconducting cavities can provide an effective and efficient means of accelerating ion beams to velocities as high as $0.7 c$.

As applied to the RIA driver linac, spoke-loaded cavities provide a number of advantages compared with the higher-frequency, elliptical-cell cavities that have been proposed for this application. In particular, using TEMclass, spoke-loaded cavities (1) reduces the required num- ber of cavities from 180 to 140; (2) reduces the number of cavity types in the high-energy section from three to two; (3) increases the operating temperature from 2 to $4.2 \mathrm{~K}$; (4) reduces the refrigeration required by approximately a factor of 2; (5) increases the longitudinal acceptance by nearly a factor of 5 , decreasing the possibilities for beam loss and activation.

In addition, TEM-class spoke-loaded cavities have excellent mechanical stability, minimizing the difficulty of tuning and phase control.

\section{ACKNOWLEDGMENTS}

The authors acknowledge many helpful discussions with numerous colleagues, most particularly with Peter Kneisel (JLAB), Jerry Nolen (ANL), Ed Peterson (AES), and Dale Schrage (LANL). This work was supported by the U.S. Department of Energy under Contracts No. DEAC05-ER40150 and No. W-31-109-ENG-38.

[1] J. R. Delayen, in Proceedings of the 1988 Linear Accelerator Conference, Williamsburg VA, 1988 (CEBAF Report No. 89-001, 1988), p. 199.

[2] J. R. Delayen, W. L. Kennedy, and C.T. Roche, in Proceedings of the 1992 Linear Accelerator Conference, Ottawa, Ontario, Canada, edited by C. R. Hoffmann (AECL Report No. 10728, 1992), p. 695.

[3] K.W. Shepard, M. Kedzie, J. R. Delayen, J. Mammosser, and C. Piller, in Proceedings of the 1999 Particle Accelerator Conference, New York, NY (IEEE Catalog No. 99CH36366, 1999), p. 955.

[4] M. White, in Proceedings of the 2002 Linear Accelerator Conference, Gyeongju, South Korea (to be published).

[5] J. A. Nolen, in Proceedings of the 2002 Linear Accelerator Conference, Gyeongju, South Korea (Ref. [4]).

[6] K.W. Shepard et al., in Proceedings of the 9th International Workshop on RF Superconductivity, Santa $\mathrm{Fe}$, New Mexico, 1999 (Los Alamos, NM, 2000), p. 345.

[7] G. Ciovati et al., in Proceedings of the 2001 Particle Accelerator Conference, Chicago, IL (IEEE Catalog No. 01CH37268, 2001), p. 484.

[8] T. Tajima et al., in Proceedings of the 2001 Particle Accelerator Conference, Chicago, IL (Ref. [7]), p. 1119.

[9] C. C. Compton et al., in Proceedings of the 2001 Particle Accelerator Conference, Chicago, IL (Ref. [7]), p. 1044.

[10] J. R. Delayen, in Proceedings of the 4th Workshop on RF Superconductivity, Tsukuba, Japan, 1989 (KEK Report No. 89-21, 1989), p. 249.

[11] K.W. Shepard, Nucl. Instrum. Methods Phys. Res., Sect. A 382, 125 (1996).

[12] J. R. Delayen, in Proceedings of the 10th Workshop on RF Superconductivity, Tsukuba, Japan, 2001 (KEK Report No. 2003-2, 2003).

[13] S.H. Kim, in Proceedings of the 2002 Linear Accelerator Conference, Gyeongju, South Korea (Ref. [4]).

[14] W. J. Schneider, P. Kneisel, and C. Rode, in Proceedings of the 2003 Particle Accelerator Conference, Portland, 
OR (Stanford Linear Accelerator Center, Stanford, CA, to be published), http://www-conf.slac.stanford.edu/ pac03/Default.htm

[15] J. Fuerst, M. Kedzie, M. Kelly, and K. Shepard, in Proceedings of the 2003 Particle Accelerator Conference, Portland, OR (Ref. [14]).

[16] CST MICROWAVE STUDIO, Computer Simulation Technology (http://www.cst.de/)

[17] Kenneth W. Shepard, in Proceedings of the 2002 Linear Accelerator Conference, Gyeongju, South Korea (Ref. [4]).

[18] I. E. Campisi et al., in Proceedings of the 2002 Linear Accelerator Conference, Gyeongju, South Korea (Ref. [4]).

[19] T. Tajima et al., in Proceedings of the 2002 Linear Accelerator Conference, Gyeongju, South Korea (Ref. [4]).

[20] W. Hartung et al., in Proceedings of the 2003 Particle Accelerator Conference, Portland, OR (Ref. [14]).

[21] R. A. Byrns and M. A. Green, Adv. Cryog. Eng. 43, 1661 (1998).

[22] V. Ganni (private communication).

[23] Hasan Padamsee, Jens Knobloch, and Tom Hays, RF Superconductivity for Particle Accelerators (J. Wiley and Sons, New York, 1998), p. 88.
[24] E. P. Knapp, in Proceedings of the 1971 Particle Accelerator Conference [IEEE Trans. Nucl. Sci. 18, 508 (1971)].

[25] S. K. Esin, L.V. Kravchuk, V. A. Matveev, P. N. Ostroumov, and V. L. Serov, in Proceedings of the 1994 Linear Accelerator Conference, Tsukuba, Japan, edited by K. Takata et al. (National Laboratory for High Energy Physics (KEK), Tsukuba, Japan, 1994), pp. 31-35.

[26] R. J. Noble, in Proceedings of the 1992 Linear Accelerator Conference, Ottawa, Ontario, Canada (Ref. [2]), p. 565.

[27] Y. Yamazaki et al., in Proceedings of the 1999 Particle Accelerator Conference, New York, NY (Ref. [3]), p. 513.

[28] P. N. Ostroumov and K. W. Shepard, Phys. Rev. ST Accel. Beams 3, 030101 (2000).

[29] P. Ostroumov et al., Phys. Rev. ST Accel. Beams 5, 060101 (2002).

[30] P. N. Ostroumov, in Proceedings of the ICFA Advanced Beam Dynamics Workshop "Halo'03," Montauk, NY, 2003 (Brookhaven National Laboratory, Upton, NY, to be published).

[31] D. Berkovits and A. Facco, LNL Report No. LNL-INFN (REP) 191/2002, 2002.

[32] J. F. Ziegler, J. P. Biersack, and U. Littmark, The Stopping and Range of Ions in Solids (Pergamon Press, New York, 1985); J. F. Ziegler, Program SRIM, IBM-Research. 